\title{
The benefits of Combined PET/CT and PET/MRI technologies for cancer care
}

\author{
Mwahib Sid Ahmed Mohamed Osman Aldosh, \\ Ph. D, Assistant Professor,Co-ordinator of Radiological Sciences Department \\ Applied Medical College - Najran University- Kingdom of Saudi Arabia
}

\begin{abstract}
Cancer is one of leading causes of morbidity and mortality in developed countries. Most radiologic procedures map the anatomy and morphology of tumors with little or no information about their metabolism .Positron emission tomography (PET) is a coalition of physics, chemistry, physiology, and medicine united in an effort to measure physiologic parameters noninvasively. The combination of PET and CT has matured into an important diagnostic tool. During the same period, concepts for PET scanners integrated into an MR tomography have emerged. The excellent soft-tissue contrast of MRI and the multifunctional imaging options it offers, such as spectroscopy, functional MRI, and arterial spin labeling, complement the molecular information of PET. These combination have many benefits for the evaluation and early detection of pathological characterization, and recognized individualized treatments, particularly for cancer disease .This evaluation can provide valuable and important aspects for improving the survival of cancer patients. Thisresearchpaper is focus ondescribing and explore the benefits of new development radiological techniques and discuss the various imaging characteristics for diagnosis and treatment of cancer ,offering great potential for improvements in the care of cancer patients in the near future.
\end{abstract}

Key Words: Cancer ; positron emission tomography ; technology ; PET/CT; PET/MRI ; oncology

\section{Introduction:}

Imaging is becoming an increasingly important tool in both research and clinical care. A range of new imaging technologies now provide unprecedented sensitivity to visualization of structure and function from the level of individual molecules to the whole body. Techniques in molecularimaging have developed from stand alone modalitiesto multimodality methods.Among these, thecombination of positron emission tomography (PET)with computed tomography (CT) and magnetic resonance imaging (MRI).combination of positron emission tomography (PET)and computed tomography (CT) is a successful imagingmethod and has become an important tool in clinical practice. Technological approaches that combine magneticresonance imaging (MRI) with positron emission tomography(PET) have now been introduced. PET/MRI and the resultingcombination of molecular, morphological and functional information will pave the way for a better understandingof physiological and disease mechanisms in preclinical and clinical settings, we describe its use in the study of cancer.

Cancer is ofgreat public health importance, with approximately 1.5 million new cases last year in the US (1). Obviously, otherfields of research such as neurology (2)and cardiology(3) also comprise fields where PET/MRI can be applied. Cancer exhibits not only a highly heterogeneous pathologywith respect to the cell type and tissue origin, but alsoinvolves multiple pathways and fundamental cell processes such as persistent growth signals, the evasion of apoptosis,insensitivity to antigrowth signals, unlimited replicative potential, angiogenesis, invasion and metastasis(4). Furthermore, changes in the cellular metabolism(5), tumor stroma, pluripotent stem cells and intercellularcommunication via gap junctions play important roles incarcinogenesis (6). Therefore, imaging techniques illuminatingthe biology and pathophysiology of cancer also haveto be sophisticated and multilayered.

\section{PET/CT technology}

Accumulation can easily be confused with normal physiologic uptake, leading to false-positive or false-negative findings. Coregistration of PET scans with CT using a combined PET-CT scanner (Figure1-1) improves the overall sensitivity and specificity of information provided by PET or CT alone . advantage is ability to correlate findings at two complementary imaging modalities in a comprehensive examination. Hence, PET-CT provides more precise anatomic definition for both the physiologic and pathologic uptake. In combined PET/CT, cross-talk effects are virtually nonexistent and CT data can beused directly for the PET attenuation correction and image reconstruction. Clinical studies have demonstrated the advantagesof PET/CT over separately performed PET and CT, itshelpful in accurate localization of small areas of increased radiotracer activity that would have been difficult or not possible to localize on PET images alone. Also it helps in distinguishing structures that normally show high metabolic activity from those with abnormally increased 
activity.PET-CT combines the advantages of the excellent functional information provided by PET and the superb spatial and contrast resolution of CT, in addition to attenuation correction for quantitative or semi quantitative assessment of data is possible by using the CT dataandthe technology has evolved rapidly into a powerful diagnostictool, particularly in the field of oncology (7).Thus, PET andCT have already proven to be ideal partners.

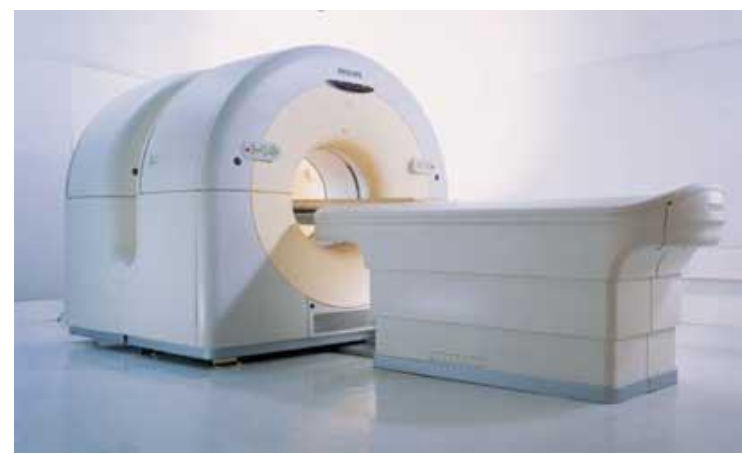

Figure 1-1 Images of CT PET scanner

\section{PET/MRI technology:}

The concept of PET is to radiolabel a bio-compound, inject it into the patient, and then measure its biodistribution as a function of time to determine physiologic quantities associated with the bio compound. All PET compounds are radiolabeled with positron-emitting radionuclides. The main principle of PET is based on the annihilationcoincidence detection of positron-emitting radionuclides.Following positron decay, two $511 \mathrm{keV}$ photons are emittedat an angle of approximately 1808 and simultaneouslydetected by scintillation crystals. The light of the scintillation

crystals is further converted into electrical signals, which are adequately processed and reconstructed to deliverimage data.By contrast, MRI exploits the magnetic properties of thehydrogen atoms in an object (human body or animal) and its interaction with an external magnetic field and radio waves.Radio waves alter the alignment of the hydrogenmagnetization and produce induced MR signals detectable by the scanner(8).

The idea to combine PET and MRI (Figure1-3) arose as early as the mid 1990s, even before PET/CT was introduced. The PET/MRI combination requires 3 risky technologic steps that modify state-of-the-art PET and MRI. First, the photomultiplier technology must be replaced with magnetic field-insensitive photodiodes . Second, compact PET detectors must be constructed so that it shouldn't interfere with the field gradients or MR radiofrequency. Finally, the MRI scanner must be adapted to accommodate the PET detectors and to allow simultaneous data acquisition without mutual interference.It is reasonable to expect that brain PET/MRI will providenew insights in the field of neuroscience and neurologicdisorders, such as neurodegeneration, brain ischemia, neurooncology,or seizures (9)It is feasible with current prototypesand future-generation systems to simultaneously study brainfunction, metabolism, oxygen consumption, and perfusion. The exact spatial and temporal coregistration of data will allowthe attribution of functional and molecular information to evenanatomically small brain structures. For the first time, it maybecome possible to study the correlation of local radiotraceruptake and brain perfusion. Time-dependant processes such asperfusion changes in stroke patients may rely on simultaneousdiffusion-weighted imaging and detection of PET perfusionto determine the optimal therapy procedure. In neurooncology, an accurate spatial match between PET and MRI data ismandatory for both radiation therapy planning and biopsyguidance. PET may detect especially small lesions with highersensitivity than MRI (Fig. 1-2) 


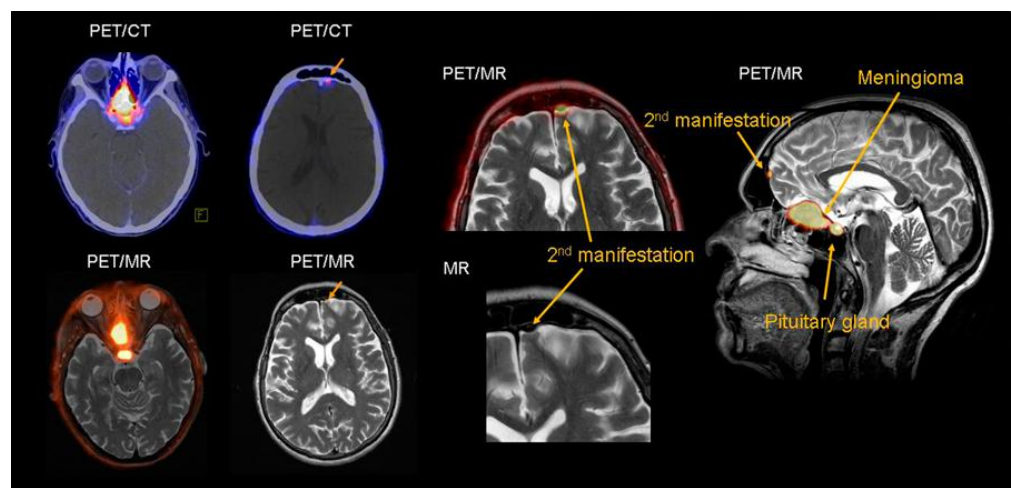

Figure1-2 PET/CT and PET/MRI of the brainshows frontobasal meningiomain olfactory region

Cancer increasingly account for morbidity and mortality.Therapeutic success with these chronic and often incurablediseases is linked to early diagnosis, accurate staging, andtherapy monitoring. This requires repeated whole-body assessmentof the extent of the disease, relapses, complications, andconcomitant diseases. Clinical studies comparing 18F-FDGPET/CT and whole-body MRI indicate that therapeuticallyrelevant information is frequently obtained by PET or MRI butnot necessarily by CT(10-14).. For example, MRI is moresensitive than PET/CT in the detection of brain, bone, and livermetastases, whereas PET/CTis more accurate in the detection oflymph node metastases, characterization soft-tissue masses, and therapy monitoring . PET/MRI could beparticularly useful for early tumor detection and functionaltherapy monitoring in oncology. It will likely be an ideal tool for investigating the effect of novel drugs, such as inhibitors ofangiogenesis or modulators of the immune system. Integratedinformation on individual cell metabolism and microenvironmentand their response to therapy will help elucidate themechanism of action and optimize treatment schedules.Real timemonitoring of the success of radiofrequency ablation byPET may be an emerging application for PET/MRI(14).

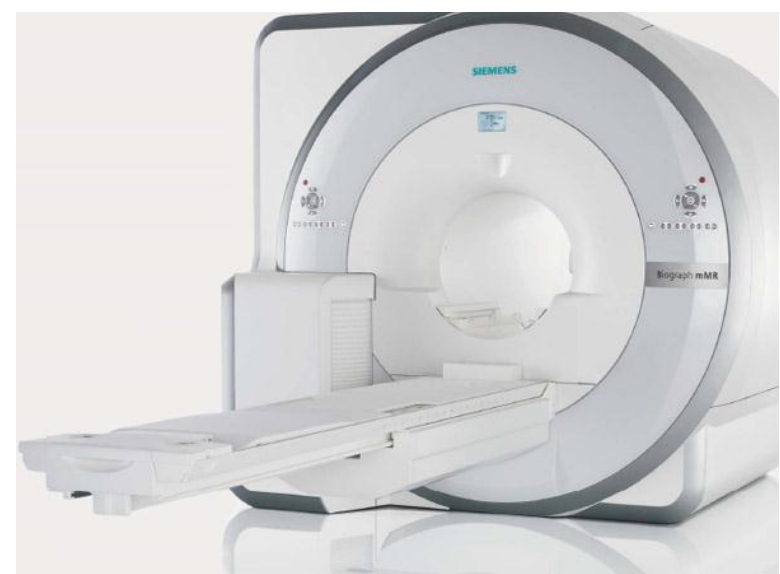

Figure 1-3 Images of MRI PET scanner

\section{Advances in oncology:}

Based on initial findings, physicians foresee the first routine applications in tumor delineation and characterization. PET/MR's ability to detect small lesions in soft-tissue is promising for diagnosis and treatment of a wide range of cancers, including those of the head and neck, the liver, lung, and prostate, as well as endometrial, ovarian, and cervical cancers.

"PET/MR is effective due to high soft-tissue contrast and the additional metabolic information, especially for primary tumor and local tumor recurrence, for example, in the liver. Overall, malignancies in the pelvic region will be a possible future application for PET/MR."

The advantage of using MR over CT for tumor detection in the liver is notable because MR is a more specific test for characterizingliver pathology than CT.(14)

Compared to individual modalities, PET/MR may pick up additional lesions in patients with extensive metastatic spread. "In patients with metastatic spread, the high sensitivity of diffusion-weighted imaging combined with PET may help identify additional lesions. it will also enhance detection of metastases in the liver, lymph nodes, and bone, as well as those in the brain and head and neck.

In patients with multiple myeloma and lymphoma, "there is the benefit of the added contrast and the bone marrow where some patients with multiple myeloma all have bone marrow involvement where CT is not 
as good as MRI in discriminating. The combination of MR and PET adds perfusion information to dynamic PET data. These techniques might play an important role for therapy adoption and when working with specific PET tracers. The advantage of the additional perfusion information is twofold: On one hand, it will be relevant in monitoring anti-angiogenic strategies. On the other, dynamic-contrast imaging plays an important role in tumor characterization and lesion detection.

\section{Conclusion:}

Having witnessed an impressive technologic developmentof PET detector technology, first PET/CT prototype systems, and MRI-based PET , advantage of PET/MRI over PET/CT is that PET/MRI not associated with significant radiation exposure, in addition to higher soft tissue contrast discrimination. Furthermore, MRI allows for additional techniques - such as angiography, functional MRI, diffusion ,spectroscopy and perfusion techniques within one single examination. PET/MRI will provide new insights in the field of neuroscience and neurologic disorders. In oncology, an accurate spatial match between PET and MRI data is mandatory for both radiation therapy planning and biopsy guidance.

results, we now seek opportunities to translate these technologic advances into clinical benefits.

\section{Acknowledgment:}

Researcher would like to extend gratitude to the Dean ship and staff of the college of Medical Radiological Sciences, Najran University and hops from Allah to make this research benefit to the development of health care all over the world, particularly for cancers patients.

\section{References:}

[1]. Antoch G, Vogt FM, Freudenberg LS, et al. Whole-body dual-modality PET/CT and whole-body MRI for tumor staging in oncology. JAMA. 2003;290:3199-3206.

[2]. Bao A, Goins B, Dodd GD III, et al. Real-time iterative monitoring of radiofrequency ablation tumor therapy with 15O-water PET imaging. J Nucl Med. 2008;49:1723-1729.

[3]. Nekolla SG, Martinez-Moeller A, Saraste A. PET and MRI in cardiac imaging: from validation studies to integrated applications. Eur J Nucl Med Mol Imaging. 2009;36(suppl 1):S121-S130.

[4]. Higuchi, T. et al. (2007) Imaging of angiogenesis in cardiology. Eur. J. Nucl. Med. Mol. Imaging. 34 (Suppl 1), S9-S19

[5]. Hanahan, D. and Weinberg, R.A. (2000) The hallmarks of cancer. Cell 100, 57-70

[6]. Tennant, D.A. et al. (2009) Metabolic transformation in cancer. Carcinogenesis 30, 1269-1280

[7]. Trosko, J.E. et al. (2004) Ignored hallmarks of carcinogenesis: stem cells and cell-cell communication. Ann. N. Y. Acad. Sci. 1028, 192-201

[8]. Mansfield, P. and Maudsley, A.A. (1976) Line scan proton spin imagingin biological structures by NMR. Phys. Med. Biol. 21, 847852) Margolis, D.J. et al. (2007) Molecular imaging techniques in bodyimaging. Radiology 245, 333-356 )

[9]. Lauenstein TC, Freudenberg LS, Goehde SC, et al. Whole-body MRI using a rolling table platform for the detection of bone metastases. EurRadiol. 2002;12:2091-2099.

[10]. Muller-Horvat C, Radny P, Eigentler TK, et al. Prospective comparison of the impact on treatment decisions of whole-body magnetic resonance imaging and computed tomography in patients with metastatic malignant melanoma. Eur $\mathbf{J}$ Cancer. $2006 ; 42: 342-350$.

[11]. Pfannenberg C, Aschoff P, Schanz S, et al. Prospective comparison of 18Ffluorodeoxyglucose positron emission tomography/computed tomography and whole-body magnetic resonance imaging in staging of advanced malignant melanoma. Eur J Cancer. 2007;43:557-564.

[12]. Schmidt GP, Baur-Melnyk A, Herzog P, et al. High-resolution whole-body magnetic resonance image tumor staging with the use of parallel imaging versus dual-modality positron emission tomography-computed tomography: experience on a 32-channel system. Invest Radiol. 2005;40:743-753.

[13]. Desar, I.M. et al. (2009) Beyond RECIST: molecular and functional imaging techniques for evaluation of response to targeted therapy. Cancer Treat. Rev. 35, 309-321

[14]. Townsend DW. Dual-modality imaging: combining anatomy and function.J Nucl Med. 2008;49:938-955. 